\title{
OCT-Documented Incision Features and Natural History of Clear Corneal Incisions Used for Bimanual Microincision Cataract Surgery
}

\author{
Graham W. Lyles, MD*, Kenneth L. Cohen, MD, and Diana Lam, BS ${ }^{\dagger}$ \\ *Department of Ophthalmology, University of North Carolina School of Medicine, Chapel Hill, NC \\ tDepartment of Biostatistics, University of North Carolina School of Public Health, Chapel Hill, NC
}

\begin{abstract}
Purpose-Use of anterior segment OCT to identify features and natural history of bimanual incisions used only for phacoemulsification and a main incision used only for intraocular lens insertion.

Methods-Prospective study of 25 subjects who had uncomplicated bimanual phacoemulsification. The Zeiss Visante OCT identified incisions at 1 day, 1 week, and 1 month postoperatively. The absence or presence of features, endothelial gaping, detachment of the Descemet membrane, endothelial misalignment, epithelial gaping, and/or loss of coaptation, was recorded. Main incision data were compared with bimanual incision data. The difference for each feature, between the main incision and the bimanual incision, at each time point and over time were calculated.
\end{abstract}

\begin{abstract}
Results-The most common findings at 1 day, 1 week, and 1 month were main incision endothelial misalignment (52\%) and bimanual incision endothelial gape (24\%), main incision endothelial misalignment (60\%) and bimanual incision endothelial gape (22\%), and main incision endothelial misalignment (35\%) and bimanual incision endothelial misalignment (14\%), respectively. Only at 1 day and 1 week was endothelial misalignment significantly more common in the main incision ( $P=0.0352$ and 0.0005 , respectively). The only other incision feature that differed significantly was endothelial gape, which was more frequent in the bimanual incision at 1 week $(P=0.0391)$. For each incision feature combined over all time periods, only endothelial misalignment was significantly more prevalent in the main incision $(P=0.0004)$, whereas endothelial gape was more common in the bimanual incision $(P=0.0352)$.
\end{abstract}

Conclusions-Using OCT, bimanual phacoemulsification did not adversely affect the anatomic incision integrity and healing.

\section{Keywords}

bimanual microincision cataract surgery; anterior segment optical coherence tomography; clear corneal incision; wound architecture; wound healing; incision integrity

The use of power modulation and the development of a new generation of intraocular lenses (IOL) have allowed phacoemulsification surgical techniques to move toward ever smaller

Copyright (C) 2011 by Lippincott Williams \& Wilkins

Reprints: Graham W. Lyles, Department of Ophthalmology, University of North Carolina School of Medicine, 5151 Bioinformatics Building, CB 7040, Chapel Hill, NC 27599-7040 (grahamlyles@ gmail.com).

No author has a financial or proprietary interest in the product, method, or material presented. 
incisions. ${ }^{1-3}$ It is now possible to perform phacoemulsification and implant IOLs through incisions less than $2 \mathrm{~mm}$, which is commonly referred to as microincision cataract surgery (MICS). ${ }^{3}$ There are 2 types of MICS phacoemulsification: bimanual and coaxial.

The bimanual MICS technique separates the irrigation and aspiration functions, each to a separate instrument. There is no irrigating sleeve on the phacoemulsification tip, which is known as sleeveless phacoemulsification. The efficacy of sleeveless phacoemulsification is established. When bimanual MICS was performed using an IOL that can be inserted through a sub-2 mm incision, visual acuities, corrected and uncorrected, were excellent, and retinal image quality was equal to that of conventional IOLs. ${ }^{3,4}$ Furthermore, bimanual MICS using sub-2 mm incision IOLs did not induce corneal astigmatism or corneal optical aberrations. ${ }^{5}$ Thus, bimanual MICS produced excellent outcomes.

Still, bimanual sleeveless MICS is criticized for its steep learning curve, anterior chamber instability, limited infusion because of small instruments, and mechanical wound trauma. ${ }^{6}$ In part, to overcome these supposed problems, coaxial phacoemulsification through a sub-2 $\mathrm{mm}$ incision was introduced, coaxial MICS. ${ }^{7}$ When directly compared, coaxial and bimanual MICS, each had excellent visual results and similar surgically induced astigmatism and corneal endothelial cell loss. ${ }^{8,9}$ One ex vivo study, using scanning electron microscopy to describe the internal configuration of bimanual MICS incisions, suggested that the bimanual MICS incisions have less integrity than the coaxial MICS incisions; however, more recent clinical studies suggest otherwise. ${ }^{8-10}$

Anterior segment optical coherence tomography (OCT) can be used to describe the incision architecture of clear corneal incisions (CCI). ${ }^{11,12}$ To help understand the clinical healing of bimanual MICS incisions, the purpose of our study is to use OCT to describe changes of incision architecture over time after bimanual sleeveless MICS.

\section{MATERIALS AND METHODS}

\section{Subjects}

This study was approved by the Institutional Review Board of the University of North Carolina School of Medicine. In this prospective observational study, 25 subjects with cataract and no other eye disease were enrolled from the Department of Ophthalmology of the University of North Carolina School of Medicine and underwent standard informed consent for participation. Exclusion criteria included those who (1) suffer from diabetes and have diabetic retinopathy, (2) have a history of intraocular surgery, (3) have a history of ocular trauma, (4) have known pathology of the cornea, (5) are unable to understand English, (6) are decisionally impaired, (7) are currently incarcerated, and/or (8) are less than 18 years of age.

\section{Examination}

All subjects underwent standard preoperative examination and evaluation by the primary investigator (K.L.C.). After bimanual MICS, subjects underwent standard sequential postoperative visits at 1 day, 1 week, and 1 month. At the conclusion of each visit, subjects underwent anterior segment OCT evaluation by the same masked examiner using the Visante AS-OCT (Carl Zeiss Meditec, Inc, Dublin, CA). At the 1-week visit, the 10-0 nylon suture was removed before anterior segment OCT evaluation.

\section{Surgical Technique}

All subjects underwent bimanual MICS as described previously with burst mode power modulation (Table 1). ${ }^{13}$ All surgery was performed by the same attending surgeon (K.L.C.), 
experienced in bimanual MICS. Topical anesthetic was used. The Duet BiManual System (MicroSurgical Technology, Redmond, WA) was used. Superotemporally and inferotemporally, $1.2 \times 1.4 \mathrm{~mm}$ trapezoidal limbal incisions, were made with a Sharpoint (Surgical Specialties Corporation, Vancouver, Canada) IQ Geometry ClearTrap Knives Bevel Up (catalog number: 77-1214). Incisions were created as described by Fine et al. ${ }^{11}$ Sodium hyaluronate $3.0 \%$ and chondroitin sulfate $4.0 \%$ (Viscoat; Alcon Laboratories, Inc, Ft Worth, TX) was injected into the anterior chamber. A continuous curvilinear capsulorrhexis was performed with a 23-gauge capsulorrhexis forceps (Fine/Hoffman DFH-0002). Hydrodissection and hydrodelineation were performed. The Infiniti phacoemulsifier (Alcon Laboratories, Inc.) was used to remove the cataract. A round 0.9$\mathrm{mm}$ turbosonics microtip (ref no: 30RTS; Alcon Laboratories, Inc) without the irrigation sleeve and a 20-gauge horizontal (Fine/Nagahara; Du-0233) or vertical (Fine/Olson; Du-02305) chopper were used to chop and emulsify the nucleus and epinucleus. Twentygauge irrigation and aspiration tips (Du-02301 and Du-02302) were used to remove the cortex. The anterior chamber was formed with Viscoat, and a $2.75-\mathrm{mm}$ temporal CCI was made using a Sharpoint clear corneal knife double bevel (catalog number: 72-2763). An AR40e IOL (Advanced Medical Optics, Santa Anna, CA) was injected into the capsular bag. Viscoat was removed by using the irrigation and aspiration tips. The $2.75-\mathrm{mm}$ incision was closed with one 10-0 nylon suture, which was removed at 1 week after surgery. Light hydration of all incisions was performed with balanced salt solution and a 27 -gauge blunttipped cannula. Incisions were visually inspected to ensure self-sealability.

\section{Image Analysis of Corneal Incisions}

A standardized protocol was employed by the examiner, which ensured that each of the 3 incisions was adequately imaged. With the subject fixated centrally, multiple images of each incision were acquired. With Visante software version 2.0.1.88, the "enhanced anterior segment single" option was chosen to capture scans of the corneal incisions. To capture the main 2.75-mm incision, a 16-mm horizontal scan was obtained. To capture each $1.2 \times 1.4$ $\mathrm{mm}$ trapezoidal incision, the $16-\mathrm{mm}$ scan was rotated approximately 60 degrees clockwise and counter clockwise. The clearest images were captured and saved for analysis.

The same examiner analyzed all the saved images. A standard pictorial grading sheet with 6 unique incision characteristics was used for evaluation (Fig. 1). Incision features counted were epithelial gape, epithelial misalignment, loss of coaptation, endothelial gape, endothelial misalignment, and detachment of the Descemet membrane. ${ }^{14}$

Because multiple images were obtained of each incision, the presence of any incision feature(s) in any one of the scans was counted as present. Multiple incision features were sometimes present in the same incision, and each feature was counted as present.

\section{Statistical Analyses}

For each incision, feature, and visit combination $(3 \times 6 \times 3)$, if there was presence of the feature, then the incision-feature-visit combination had a record of 1 . For example, if there was endothelial misalignment in the main incision during the first visit, then the variable for (main, visit 1, endothelial misalignment) would have a 1 recorded for that subject.

Otherwise, a 0 was recorded. The data for the inferior and superior bimanual incisions were combined by taking the mean of the 2 incisions for each feature-visit combination. The combination of the inferior and superior incision is referred to as the bimanual incision. The difference for each feature between the main incision and the bimanual incision at each time point (1 day, 1 week, and 1 month) was postoperatively calculated. Differences over all time points were calculated by averaging the mean of differences over time and incision, 
respectively. The Mantel-Haenszel test was used to determine if there was a difference between the main incision and the bimanual incision over time points.

Subject demographic variables were tested to determine if the study was balanced. The sign test was performed to compare groups. The $P$ values were based on testing the null hypothesis that the proportion of men/women and right/left eyes is equal to 0.5 .

\section{RESULTS}

The mean (SD) age of subjects was 73.4 years (9.1). Sixteen subjects (64\%) were women; 9 subjects $(36 \%)$ were men. Fourteen right eyes $(56 \%)$ and 11 left eyes $(44 \%)$ were analyzed. There were no significant differences in the proportions of men and women $(P=0.1615)$ nor right and left eyes $(P=0.5484)$ (Table 2). Data from 25 subjects were available at 1 day and 1 week; data from 23 subjects were available at 1 month.

Data from the main incision (used only for IOL injection) were compared with the bimanual incision (which was used only for bimanual phacoemulsification). Bar graphs were created showing the percent of each incision feature, at each time period, for the main incision and the bimanual incision, respectively (Fig. 2). The most common findings at 1 day were endothelial misalignment in the main incision (52\%) and endothelial gape in the bimanual incision (24\%). The most common findings at 1 week were endothelial misalignment in the main incision (60\%) and endothelial gape in the bimanual incision (22\%), similar to the pattern seen at 1 day. The most common finding at 1 month was endothelial misalignment in both the main incision (35\%) and the bimanual incision (14\%).

Statistical comparison of incision features between the main incision and the bimanual incision is listed in Table 3. A $P$ value less than 0.05 was considered statistically significant. Endothelial misalignment was significantly more common in the main incision compared with the bimanual incision only at 1 day $(P=0.0352)$ and 1 week $(P=0.0005)$. The only other incision feature found to differ significantly was endothelial gape, which was more frequent in the bimanual incision compared with the main incision at 1 week $(P=0.0391)$. Missing $P$ values in Table 3 from the other incision features are because of all differences being 0 .

When each incision feature was combined over all time periods, endothelial misalignment was significantly more prevalent in the main incision compared with the bimanual incision $(P=0.0004)$, whereas endothelial gape was more common in the bimanual incision compared with the main incision $(P=0.0352)$. When all incision features were combined over all time periods, incision features were significantly more prevalent in the main incision $(P=0.0169)$.

\section{DISCUSSION}

A major advancement in cataract surgical technique is the decrease in the size of the CCIs through which the surgery is performed. Clinical studies comparing phacoemulsification through incisions less than $2 \mathrm{~mm}$, bimanual, and $2.75 \mathrm{~mm}$ and $3.00 \mathrm{~mm}$, coaxial, documented similar clinical outcomes. ${ }^{14,15}$ Although comparative studies demonstrate the clinical efficacy of bimanual phacoemulsification, there is continuing criticism of this technique. Recent criticism revolves around the anatomical integrity of CCIs, less than 2 $\mathrm{mm}$, used for bimanual phacoemulsification.

One study using cadaver eyes concluded that the incision integrity after bimanual phacoemulsification was inferior to that after coaxial phacoemulsification. ${ }^{10}$ This was based on scanning electron microscopy of only 1 eye after bimanual surgery, which had greater 
endothelial cell loss and tearing of the Descemet membrane compared with only 2 eyes after coaxial surgery. Therefore, based on a limited number of cadaver eyes, this study speculated that these findings were because of tight incision geometry surrounding the instruments. ${ }^{10}$ In our opinion, criticism of a surgical technique based on such a small sample of cadaver eyes is not justified. Also, in this study, phacoemulsification was simply simulated; crystalline lenses were not actually removed, and there was no insertion of an IOL. Finally, it is not stated whether the operating author had prior experience in using bimanual MICS. The surgeon in our study is experienced in bimanual MICS.

Subsequently, 2 studies seemed to confirm the inferior incision integrity of bimanual surgery. ${ }^{16,17}$ In rabbit eyes, after bimanual surgery, there was more irregularity to the incision margins, more stromal swelling, and more disruption of stromal collagen fibers than after coaxial surgery. Importantly, to our knowledge, there is only 1 clinical study that casts light on the incision integrity of CCIs used for bimanual phacoemulsification versus coaxial phacoemulsification. ${ }^{17}$ This study found greater ingress of trypan blue into the anterior chamber in the bimanual eyes versus the microcoaxial and the coaxial eyes. The authors suggested that, while it is tempting to perform surgery through incisions as small as possible, surgeons should minimize incision distortion as it could jeopardize incision integrity.

However, using OCT to demonstrate anatomical incision integrity and healing over time, our study does not support this statement. Our study documented and compared findings of anatomical incision integrity over time of both CCIs used only for bimanual phacoemulsification and the CCIs used only for IOL injection. Thus, importantly, the bimanual CCIs were not altered for IOL injection as in the clinical study, which evaluated trypan blue ingress into the anterior chamber. ${ }^{17}$

Figure 2 lists the prevalence of the incision features in the main and bimanual incisions. At 1 day after surgery, the most prevalent finding on OCT in all incisions was endothelial misalignment, $26.7 \%$, followed by endothelial gape at $20 \%$. All other findings were present in less than $10 \%$ of all incisions. Importantly, over time, all findings, except endothelial misalignment $(21.7 \%)$, decreased to a prevalence of less than $3 \%$ at 1 month after surgery. At 1 month after surgery, there was no Descemet detachment, loss of coaptation, epithelial misalignment, or epithelial gape. Therefore, when all incisions were combined, the most common defect of anatomic integrity was located posteriorly. This is similar to the findings after bimanual MICS in cadaver eyes and rabbit eyes. ${ }^{10,16}$

One notable aspect of our study was that the $1.2 \times 1.4 \mathrm{~mm}$ trapezoid CCIs were used only for bimanual phacoemulsification and not enlarged for IOL insertion. At conclusion of phacoemulsification, the bimanual incisions are rounded because of the configuration of the instruments used to emulsify the nucleus and epinucleus and to remove the cortex, as well as instrument maneuvers in a relatively tight incision. Therefore, enlarging a rounded incision would likely compromise sealability. To our knowledge, ours is the only study that can compare anatomic incision integrity and healing of bimanual incisions documented on OCT to the main incision used only for IOL insertion. At 1 day and 1 week, there were differences between the main incision and the bimanual incision. At 1 day, the main incision had a higher incidence of endothelial misalignment, possibly because of suture tension on the anterior portion of the incision. At 1 week, the bimanual incision had a higher incidence of endothelial gape, probably because of the tight geometry of 20-gauge bare metal instruments passing through this incision. ${ }^{17}$

These findings were short lived because at 30 days after surgery, there were no differences between the main incision and the bimanual incision (Table 3). Notably, incision features 
seemed to resolve over time in all incisions, with the exception of endothelial misalignment, which persisted in all incisions (Fig. 2).

Therefore, when compared with the main incision, bimanual MICS did not have an adverse effect on the anatomic integrity and healing of bimanual incisions. Also, suturing the main incision did not cause a difference in healing between the main incision and the bimanual incision. There are several clinical studies that used OCT to image CCIs. ${ }^{11-13,18-22}$ Only 3 documented healing over time, and only 2 documented healing to 1 month. ${ }^{20-22}$ Two studies evaluated anatomic incision integrity in bimanual MICS, and only 1 of these studies evaluated anatomic CCI integrity at 1 month. ${ }^{21,22}$ However, unlike our study, these bimanual CCIs were enlarged to accommodate IOL insertion.

The anatomic integrity of the main CCIs in our study has some findings similar to the findings in the 2 studies that had similarly sized coaxial CCIs. ${ }^{19,20}$ At postoperative day 1 , epithelial integrity was not a problem, but the incidence of faulty endothelial apposition was approximately $45 \%$ to $50 \%$. In our study, endothelial misalignment was $52 \%$ (Fig. 2). However, endothelial gape and Descemet detachment were only $12 \%$ and $4 \%$, respectively (Fig. 2). This is less than previously reported for coaxial CCIs, up to $50 \%$ for endothelial gape and $67 \%$ for Descemet membrane detachment. ${ }^{19,20}$ Thus, the main incision in our study had less disruption of the anatomic integrity of the posterior cornea, possibly because of the use of the CCIs in our study only for IOL insertion instead of both phacoemulsification and IOL insertion. Similar to our study, faulty endothelial apposition was less frequent at 1 month postoperatively (Fig. 2). ${ }^{20}$

A recent study compared the anatomic integrity of microcoaxial CCIs with bimanual CCIs and found no differences between these incisions over the first postoperative month. ${ }^{22}$ This is confirmed by our study that found for each incision feature over time no difference between the main incision and the bimanual incision. Also, this recent study found that the bimanual CCIs had more frequent endothelial gaping and Descemet membrane detachment, $64 \%$ and $60 \%$, respectively, at postoperative day 1 than our study, and this may be because of the enlargement of CCIs for IOL insertion (Fig. 2). ${ }^{22}$ Importantly, similar to our study, at 1 month, the bimanual CCIs healed well with resolution of the faulty posterior apposition.

Because our study found essentially no differences in anatomic integrity and healing when the main incision was compared with the bimanual incision and because the main incision was used only for IOL insertion, the tight incision geometry of the bimanual incisions might have affected OCT findings at 1 week, but healing over 30 days was not affected. Also, our results confirm the recent study that found little OCT-documented differences of CCIs over the 30 days after surgery from eyes that had microcoaxial phacoemulsification compared with eyes that had bimanual MICS. ${ }^{22}$ This casts doubt on the suggested adverse affect of the tight incision geometry of bimanual incisions. ${ }^{10,16,17}$ Also, even though our main incision, used only for IOL insertion, may have had less trauma than coaxial CCIs (used for both phacoemulsification and insertion of the IOL) the main incision in our study had similar incision integrity over time as coaxial CCIs. ${ }^{22}$ Thus, the absence of a sleeve and the different maneuvers required with bimanual versus coaxial phacoemulsification do not seem to adversely affect the anatomic incision integrity and healing as documented with OCT.

\section{Acknowledgments}

Supported by Research to Prevent Blindness, NY, and National Institutes of Health/National Institute of Environmental Health Sciences T32 ES007018. 


\section{References}

1. Fine IH, Packer M, Hoffman RS. Power modulation in new phacoemulsification technology: improved outcomes. J Cataract Refract Surg. 2004; 30:1014-1019. [PubMed: 15130637]

2. Donnenfeld ED, Olson RJ, Solomon R, et al. Efficacy and wound-temperature gradient of WhiteStar phacoemulsification through a 1.2 mm incision. J Cataract Refract Surg. 2003; 29:1097-1100. [PubMed: 12842674]

3. Alio JL, Rodriguez-Prats JL, Vianello A, et al. Visual outcome of microincision cataract surgery with implantation of an Acri. Smart lens. J Cataract Refract Surg. 2005; 31:1549-1556. [PubMed: 16129290]

4. Alio JL, Schimchak P, Montes-Mico R, et al. Retinal image quality after microincision intraocular lens implantation. J Cataract Refract Surg. 2005; 31:1557-1560. [PubMed: 16129291]

5. Elkady B, Alio JL, Ortiz D, et al. Corneal aberrations after microincision surgery. J Cataract Refract Surg. 2008; 34:40-45. [PubMed: 18165079]

6. Weikert MP. Update on bimanual microincisional cataract surgery. Curr Opin Ophthalmol. 2006; 17:62-67. [PubMed: 16436926]

7. Lee KM, Kwon HG, Joo CK. Microaxial cataract surgery outcomes: comparison of $1.8 \mathrm{~mm}$ system and $2.2 \mathrm{~mm}$ system. J Cataract Refract Surg. 2009; 35:874-880. [PubMed: 19393887]

8. Wilczynski M, Supady E, Piotr L, et al. Comparison of surgically induced astigmatism after coaxial phacoemulsification through $1.8 \mathrm{~mm}$ microincision and bimanual phacoemulsification through 1.7 mm microincision. J Cataract Refract Surg. 2009; 35:1563-1569. [PubMed: 19683153]

9. Wilczynski M, Supady E, Loba P, et al. Comparison of early corneal endothelial cell loss after coaxial phacoemulsification through $1.8 \mathrm{~mm}$ microincision and bimanual phacoemulsification through $1.7 \mathrm{~mm}$ microincision. J Cataract Refract Surg. 2009; 35:1570-1574. [PubMed: 19683154]

10. Berdahl JP, DeStafeno JJ, Kim T. Corneal wound architecture and integrity after phacoemulsification. Evaluation of coaxial, microincision coaxial, and microincision bimanual techniques. J Cataract Refract Surg. 2007; 33:510-515. [PubMed: 17321403]

11. Fine IH, Hoffman RS, Packer M. Profile of clear corneal cataract incisions demonstrated by optical coherence tomography. J Cataract Refract Surg. 2007; 33:94-97. [PubMed: 17189800]

12. Schallhorn JM, Tang M, Li Y, et al. Optical coherence tomography of clear corneal incisions for cataract surgery. J Cataract Refract Surg. 2008; 34:1561-1565. [PubMed: 18721720]

13. Mathys KC, Cohen KL, Armstrong BD. Determining factors for corneal endothelial cell loss by using bimanual microincision phacoemulsification and power modulation. Cornea. 2007; 26:1049-1055. [PubMed: 17893532]

14. Calladine D, Packard R. Clear corneal incision architecture in the immediate postoperative period evaluated using optical coherence tomography. J Cataract Refract Surg. 2007; 33:1429-1435. [PubMed: 17662437]

15. Alio J, Rodriguez-Prats JL, Galal A, et al. Outcomes of microincision cataract surgery versus coaxial phacoemulsification. Ophthalmology. 2005; 112:1997-2003. [PubMed: 16183129]

16. Kurz S, Krummenauer F, Gabriel P, et al. Biaxial microincision versus coaxial small-incision clear cornea cataract surgery. Ophthalmology. 2006; 113:1818-1826. [PubMed: 17011959]

17. Kaid Johar SR, Vasavada AR, Praveen MR, et al. Histomorphological and immunofluorescence evaluation of bimanual and coaxial phacoemulsification incisions in rabbits. J Cataract Refract Surg. 2008; 34:670-676. [PubMed: 18361992]

18. Praveen MR, Vasavada AR, Gajjar D, et al. Comparative quantification of ingress of trypan blue into the anterior chamber after microcoaxial, standard coaxial, and bimanual phacoemulsification. Randomized clinical trial. J Cataract Refract Surg. 2008; 34:1007-1012. [PubMed: 18499010]

19. Behrens A, Stark WJ, Pratzer KA, et al. Dynamics of small-incision clear corneal wounds after phacoemulsification surgery using optical coherence tomography in the early postoperative period. J Refract Surg. 2008; 24:46-49. [PubMed: 18269148]

20. Torres LF, Saez-Espinola F, Colina JM, et al. In vivo architectural analysis of $3.2 \mathrm{~mm}$ clear corneal incisions for phacoemulsification using optical coherence tomography. J Cataract Refract Surg. 2006; 32:1821-1826. 
21. Dupont-Monod S, Labbe A, Fayol N, et al. In vivo architectural analysis of clear corneal incisions using anterior segment optical coherence tomography. J Cataract Refract Surg. 2009; 35:444-450. [PubMed: 19251136]

22. Elkady B, Pinero D, Alio JL. Corneal incision quality: Microincision cataract surgery versus microcoaxial phacoemulsification. J Cataract Refract Surg. 2009; 35:466-474. [PubMed: 19251139] 

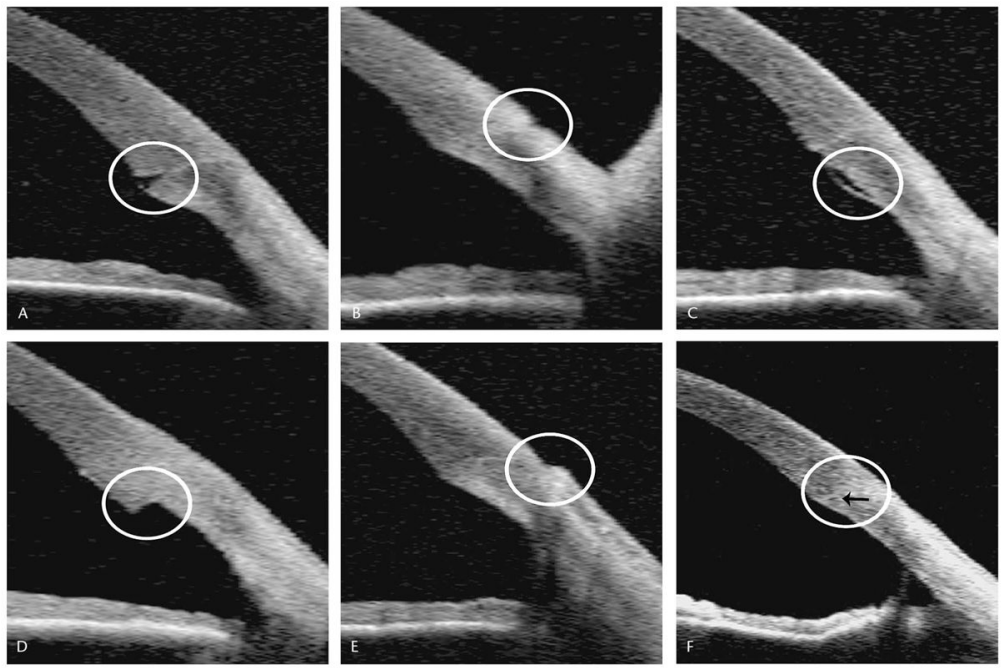

\section{FIGURE 1.}

Unique corneal incision features were visualized with anterior segment OCT. These included (circled from left to right): (A, B, C) endothelial gape, epithelial gape, and detachment of the Descemet membrane and (D, E, F) endothelial misalignment, epithelial misalignment, and loss of coaptation (arrow). 


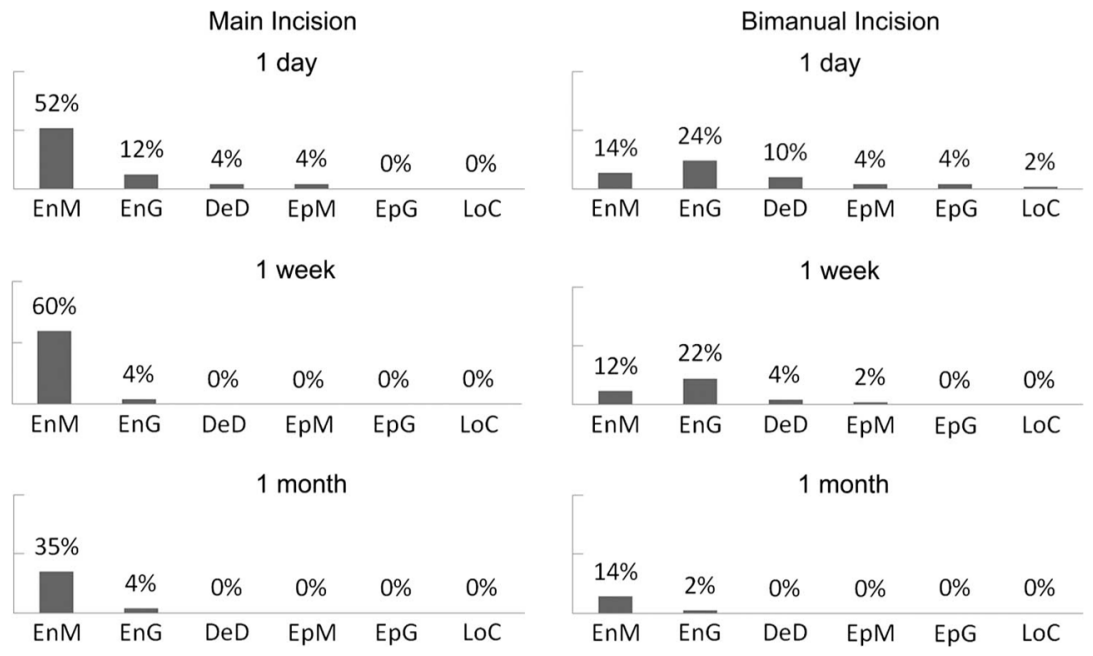

FIGURE 2.

Prevalence (\%) of each incision feature type [endothelial misalignment (EnM), endothelial gape (EnG), detachment of the Descemet membrane (DeD), epithelial misalignment (EpM), epithelial gape (EpG), and loss of coaptation (LoC)] in the main and bimanual incision at 1 day $(\mathrm{n}=25), 7$ days $(\mathrm{n}=25)$, and 30 days $(\mathrm{n}=23)$ postoperatively. 
TABLE 2

Subject Demographics

\begin{tabular}{lr}
\hline Sex, n (\%) & \\
Male & $9(36)$ \\
Female & $16(64)$ \\
Eyes, n (\%) & \\
Right & $14(56)$ \\
Left & $11(44)$ \\
Age, yrs, mean (SD) & $73.4(9.1)$ \\
\hline
\end{tabular}

Cornea. Author manuscript; available in PMC 2013 March 06. 


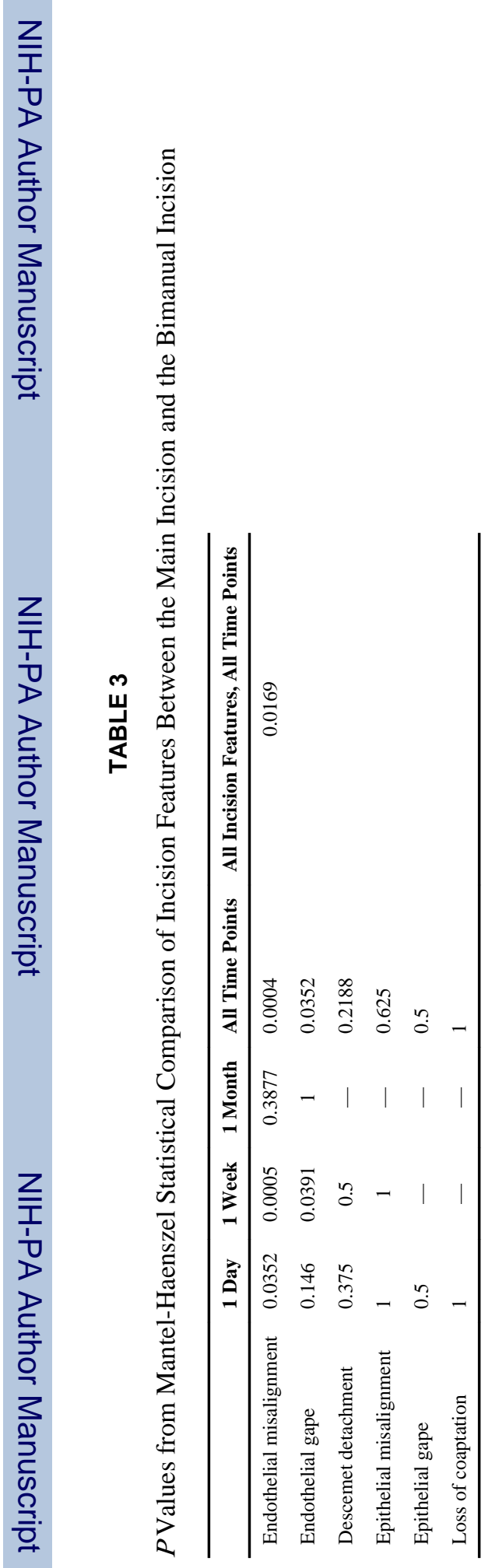

